\title{
CONCAVE HULL: A $K$-NEAREST NEIGHBOURS APPROACH FOR THE COMPUTATION OF THE REGION OCCUPIED BY A SET OF POINTS
}

\author{
Adriano Moreira and Maribel Yasmina Santos \\ Department of Information Systems, University of Minho \\ Campus de Azurém, Guimarães, Portugal \\ adriano@dsi.uminho.pt,maribel@dsi.uminho.pt
}

\begin{abstract}
Keywords: $\quad$ Concave hull, convex hull, polygon, contour, $k$-nearest neighbours.
Abstract: $\quad$ This paper describes an algorithm to compute the envelope of a set of points in a plane, which generates convex or non-convex hulls that represent the area occupied by the given points. The proposed algorithm is based on a $k$-nearest neighbours approach, where the value of $k$, the only algorithm parameter, is used to control the "smoothness" of the final solution. The obtained results show that this algorithm is able to deal with arbitrary sets of points, and that the time to compute the polygons increases approximately linearly with the number of points.
\end{abstract}

\section{INTRODUCTION}

The automatic computation of a polygon that encompasses a set of points has been a topic of research for many years. This problem, identified as the computation of the convex hull of a set of points, has been addressed by many authors and many algorithms have been proposed to compute the convex hull efficiently (Graham, 1972; Jarvis, 1973; Preparata, 1977; Eddy, 1977). These algorithms compute the polygon with the minimum area that includes all the given points (or minimum volume when the points are in a three-dimensional space). In this context, given a set of points, there is a single solution for the convex hull.

For certain applications, however, the convex hull does not represent well the boundaries of a given set of points. Figure 1 shows one example. In this example, where the points could represent trees in a forest, the region defined by the convex hull does not represent the region occupied by the trees.

This same problem, or similar problems, has already been addressed by other authors (e.g. (Edelsbrunner, 1983; Galton, 2006; Edelsbrunner, 1992a; Edelsbrunner, 1992b; Amenta, 1998)). In (Edelsbrunner, 1983) the concept of alpha-shapes was introduced as a solution to this same problem. The concept of alpha-shape was further developed in (Edelsbrunner, 1992a; Edelsbrunner, 1992b) and other solutions, such as crust algorithms (Amenta, 1998), were also proposed. However, most of the proposed approaches address the reconstruction of surfaces from sets of points, belonging to that surface and, therefore, are not optimized for the referred problem.

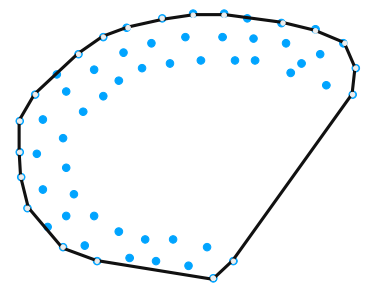

Figure 1: The area of the convex hull does not represent the area occupied by the set of points.

In other words, little work was devoted to the problem described in this paper, as also recognized by Galton et al. (Galton, 2006). In their paper, Galton et al. describe this same problem and present a few examples of applications that could benefit from a general solution to compute what they call the "footprint" of a set of points. They also describe the existing approaches, including the Swinging Arm algorithm (SA), and define a criterion with 9 "concerns" to evaluate those solutions. We therefore refer to this paper for a description of previous work on this subject. 
This paper also addresses this problem, by proposing a new algorithm for the computation of a polygon that best describes the region occupied by a set of points.

The algorithm described in this paper was developed within the context of the LOCAL project (LOCAL, 2006) as part of a solution for a broader problem. The LOCAL project aims to conceive a framework to support the development of locationaware applications, and one of its objectives is to develop a process to automatically create and classify geographic location-contexts from a geographic database (Santos, 2006). As part of this process, we faced the problem of identifying the "boundaries" of a set of points in the plane, where the points represent Points Of Interest (POIs).

In order to solve this problem, we developed a new algorithm to compute a polygon representing the area occupied by a set of points in the plane. This new algorithm filled the needs of our research project and, we believe, can be used in similar situations where the assignment of a region to a set of points is required.

This paper is organized as follows: section 2 presents the problem of creation of polygons given a set of points. Section 3 describes the Concave Hull algorithm developed for the computation of polygons with convex and non-convex shapes. Section 4 introduces the implementation undertaken, presents some examples of the obtained results, and discusses performance issues through numerical evaluation of the implemented algorithm. Section 5 concludes with some remarks and future work.

\section{COMPUTING REGIONS' BOUNDARIES}

The problem we faced in the LOCAL project was how to calculate the boundary of a geographic area defined by a set of points in the geographic space. These points represent POIs which are a common part of geographic databases and navigation systems. Figure 2 shows an example of an artificial set of POIs within a given geographic area. In this data set, one (we, humans) can easily identify 7 different regions, in addition to a number of "noise" points.

Our goal in the LOCAL project was to automatically detect these regions, while removing the noise points, and calculate the polygons that define the respective boundaries. The final result should be as shown in Figure 3.

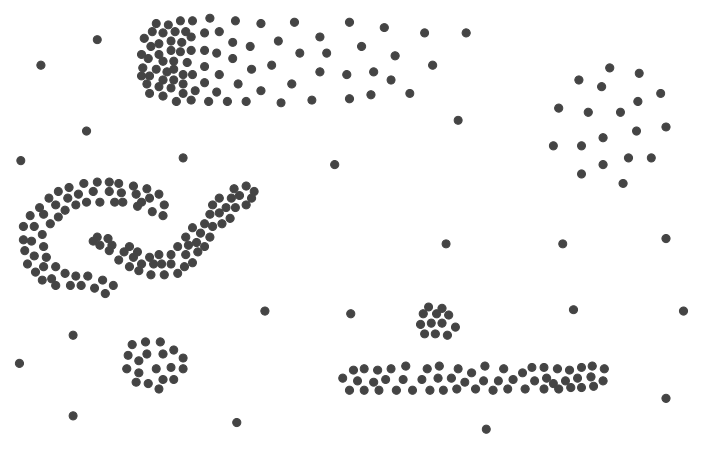

Figure 2: Initial data set.

The approach we adopted to achieve the goal depicted in Figure 3 was to divide the identification of the groups of points (identification of the points belonging to each region and noise removal), from the calculation of the polygons describing those regions, as described in (Santos, 2006), and also as suggested in (Galton, 2006).

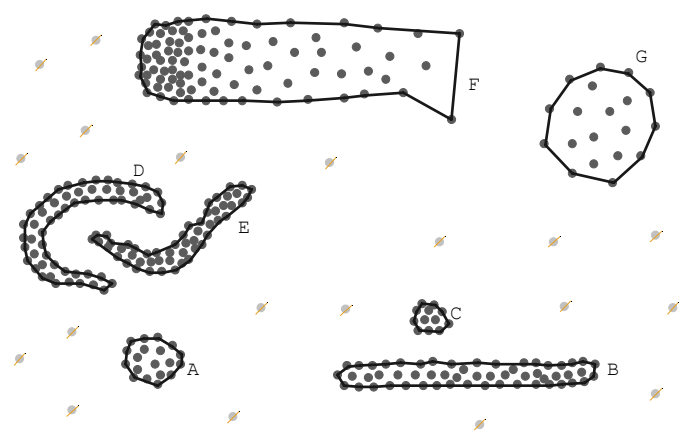

Figure 3: The goal.

For the first phase, an implementation of the Shared Nearest Neighbours (SNN) clustering algorithm was used (Ertoz, 2003). The SNN is a density-based clustering algorithm that has as its major characteristic the fact of being able to detect clusters of different densities, while being able to deal with noise and with clusters with non-globular (and non-convex) shapes. SNN uses an input parameter, $k$, which can be used to control the granularity of the clustering process. The groups of points depicted in Figure 3 were obtained using SNN with $k=8$. The noise points were also discarded by SNN (slashed points in Figure 3). In this example, the task of SNN was easy, as the seven groups of points are clearly separated from the noise points. However, with real POIs, the regions might not be so clearly defined and the regions might be of very "strange" shapes.

The second phase of the process is, for each group of points found by $\mathrm{SNN}$, to compute the 
corresponding polygon that defines the boundaries of the region. In this data set there are two distinct types of regions: the "circle shaped" regions (A, C and $\mathrm{G})$, and the other regions with less regular shapes (B, D, E and F). For the first group, there are a set of algorithms that could be used to calculate the convex hull of the points. However, for the other group of regions, the convex hull approach is not clearly a good solution, as shown in Figure 1 for the D region.

In the next section we describe the solution that was developed to overcome the limitations of the convex hull approach for this kind of applications.

\section{THE CONCAVE HULL ALGORITHM}

The goal of the algorithm described in this section is, given an arbitrary set of points in a plane, to find the polygon that best describes the region occupied by the given points. While there is a single solution for the convex hull of a set of points, the same is not true for the "concave hull". In the statement that defines our goal (previous paragraph), the expression "best describes" is ambiguous, as the "best" might depend on the final application. As an example, consider the two polygons shown in Figure 4, which describe the region E. Which of the two polygons, a) or b), "best describes" region E?

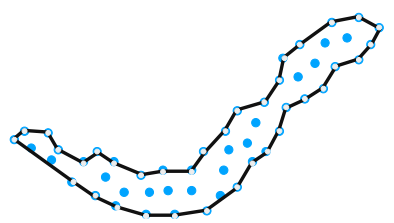

a)

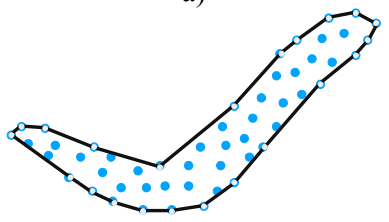

b)

Figure 4: Which one is the best? Two polygons for the same set of points.

Since there are multiple solutions (polygons) for each set of points, and the "best" solution depends on the final application, our approach to compute the polygons should be flexible enough to allow the choice of one among several possible solutions for the set of points. The other implication of this ambiguous definition of "best" is that it turns very difficult to assess the correctness of any algorithm used to compute the polygon, and even to compare different algorithms. For this last purpose, we will adopt the criteria described in (Galton, 2006) (see Section 4).

\section{1 $k$-Nearest Neighbours Approach}

Our approach to calculate the Concave Hull of a set of points is inspired in the Jarvis' March (or "gift wrapping") algorithm used in the calculation of the convex hull (Jarvis, 1973). In this algorithm, the convex hull is calculated by finding an extreme point, such as the one with lowest value of Y (in the yy axis), and then by finding the subsequent points by "going around" the points - the next point is the one, among all the remaining points, that is found to produce the largest right-hand turn.

The approach adopted for the calculation of the concave hull is similar, except that only the $k$-nearest neighbours of the current point (last founded vertex) are possible candidates to become the next point in the polygon. Figure 5 illustrates this concept.

The first step of the process is to find the first vertex of the polygon (point $A$ in Figure 5a) as the one with the lowest $\mathrm{Y}$ value. In the second step, the $k$ points that are nearest to the current point are selected as candidates to be the next vertex of the polygon (points $\mathrm{B}, \mathrm{C}$ and $\mathrm{D}$ in Figure $5 \mathrm{a}$, for $k=3$ ). In this case, point $\mathrm{C}$ is selected as the next vertex of the polygon, since it is the one that leads to the largest right-hand turn measured from the horizontal line ( $x x$ axis) that includes the first point (point A). Since $\mathrm{C}$ is now a vertex of the polygon (as well as $\mathrm{A}$ ), it must be removed from subsequent steps while searching for the $k$-nearest neighbours.

In the third step, the $k$-nearest points of the current point (point $C$ ) are selected as candidates to be the next point of the polygon (points $\mathrm{B}, \mathrm{D}$ and $\mathrm{E}$ in Figure $5 \mathrm{~b}$ ). In this case, the point that results in the largest right-hand turn, corresponding to the largest angle between the previous line segment and the candidate line segment, is selected (point E). As before, point $\mathrm{E}$ is now part of the polygon and will never be considered in the next steps.

The process is repeated until the selected candidate is the first vertex. For the first vertex (point A) to be elected as a candidate, it must be inserted again into the data set after the first four points of the polygon are computed (before that, if the first point is selected as the best candidate, a triangle is computed). By the end of the process, the polygon is closed with the first and the last point being the same (point A). 


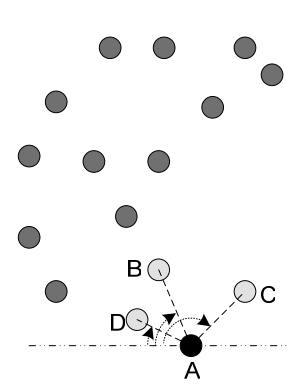

a)

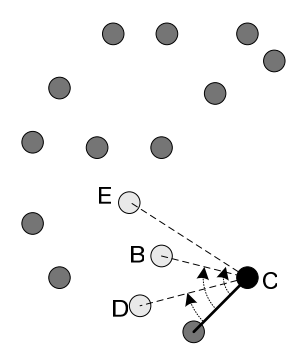

b)
Figure 5: The $k$-nearest neighbours approach.

In this example, three candidates were considered in each step $(k=3)$. If a larger number of candidates were considered, the computed polygon would become "smoother". The number of neighbours cannot, however, be larger than the number of remaining points in each step. If, in a particular step, the number of remaining points (candidates) is smaller than $k$, then the algorithm automatically considers all the remaining points (without any user intervention).

This approach works for the majority of the cases. However, there are two special cases that must be pointed out. One of them is when the selected candidate results in a polygon edge that intersects one of the already computed edges. This case is depicted in Figure 6a. In this example (step 5 ), the candidate that results into the largest righthand turn is point $\mathrm{B}$. However, this candidate leads to a polygon edge that intersects one of the existing edges and, therefore, should be discarded. In cases like this, the next candidate should be considered (point $\mathrm{G}$ in this example). If none of the candidate points (the $k$-nearest neighbours) is acceptable, then a higher number of neighbours must be considered, by increasing the value of $k$ and starting again.

The other special case may occur when the spatial density of the initial set of points is not uniform. Figure $6 \mathrm{~b}$ illustrates this case with a set of points where there are clearly two different "regions". This case should not be very common if the initial data set has gone through the clustering process (e.g using SNN), since, in that case, this data set would be separated into two different clusters. Anyway, in an arbitrary data set this special situation may occur and must be addressed.

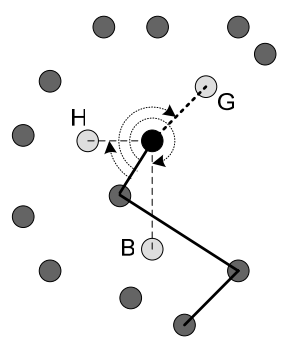

a)

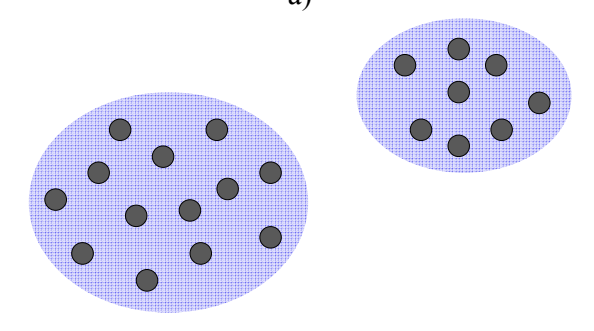

b)

Figure 6: Special cases: a) where the new edge intersects another existing edge of the polygon; b) where the points are not uniformly distributed in the space.

In this second case, the first point of the polygon is in the lower-left region (the point with the lowest Y value) and, therefore, the process starts by looking for candidates that are near this first point. However, since the points in the upper-right group are too far away from the points in the lower-left group, they are never considered as candidates if the number of neighbours (value of $k$ ) considered in each step of the process is small. As a consequence, the points in the upper-right group are left out of the polygon. To solve this issue, a higher number of neighbours must be considered. Since the value of $k$ chosen by the user might be too small, the algorithm must verify, at the end, that all the points are within the generated polygon. If not, a higher value of $k$ is automatically tried by the algorithm using a recursive process that stops when all the points are within the computed polygon.

\subsection{Concave Hull Algorithm}

The steps behind the Concave Hull concept described in the previous section were used to develop the algorithm that is shown on the next page (Algorithm 1). 
Algorithm 1: The Concave Hull algorithm.

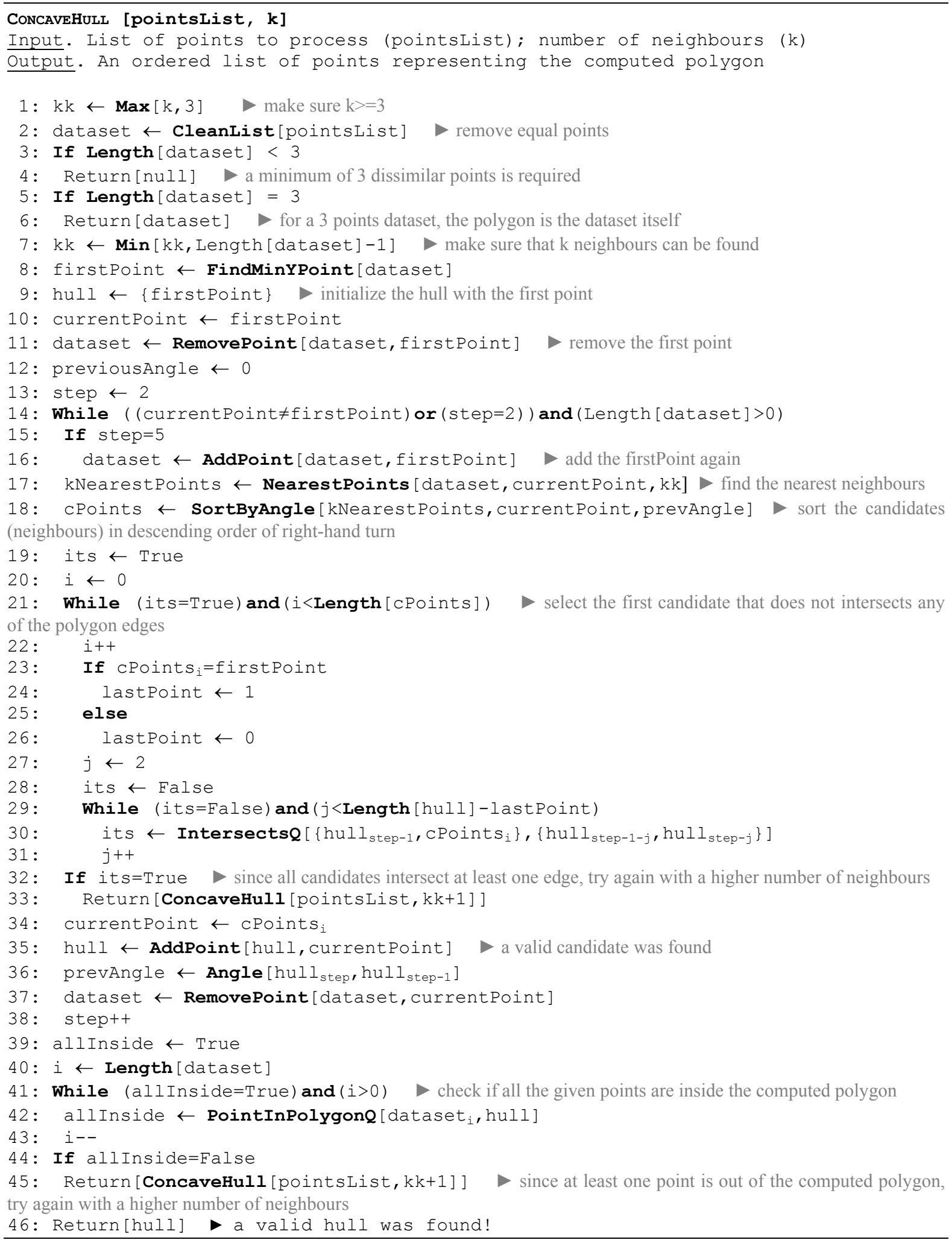


This algorithm makes use of the following functions:

CleanList[listofPoints]: returns the given listofPoints with no more than one copy of each point (removes duplicates).

Length [vector]: returns the number of elements of the given vector.

FindMinYPoint [listofPoints]: returns the element $(\{\mathrm{x}, \mathrm{y}\}$ pair) of the given listofPoints with smaller value of $Y$.

RemovePoint[vector,e]: returns the given vector without the given element $\mathbf{e}$.

AddPoint [vector, e]: returns the given vector with the given element $e$ appended as the last element.

NearestPoints [listofPoints, point, $\mathrm{k}$ ]: returns a vector with the $\mathbf{k}$ elements of listofPoints that are closer to the given point. In the current implementation, this function uses the Euclidean distance to select the nearest points. However, the distance functions can be used. This function internally re-computes the value of $k$ as the minimum value between the given value of $\mathbf{k}$ and the number of points present in the dataset.

SortByAngle [listofPoints, point, angle]: returns the given listofPoints sorted in descending order of angle (right-hand turn). The first element of the returned list is the first candidate to be the next point of the polygon.

IntersectQ [ lineSegment1, lineSegment2] : returns True if the two given lines segments intersect each other, and False otherwise.

PointInPolygonQ [point, listofPoints]: returns True if the given point is inside the polygon defined by the given listofPoints, and False otherwise.

\section{IMPLEMENTATION AND RESULTS}

The algorithm described in section 3 was implemented as a Mathematica (Mathematica, 2006) package, which was used to evaluate the algorithm and also as a tool to fulfil our project needs. In the following subsections we present a few examples of the hulls computed by this algorithm, as well as some results on its performance. The developed code (one Mathematica package) is available online on the web site of the LOCAL project, where the algorithm can be tried through a web interface.

\subsection{Results}

The polygons shown in Figure 3 (section 2) and Figure 4 (section 3) were all computed using the algorithm described in this paper. In Figure 7 two other examples are presented.

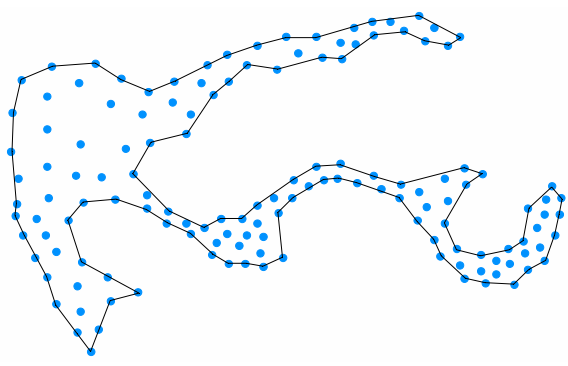

a)

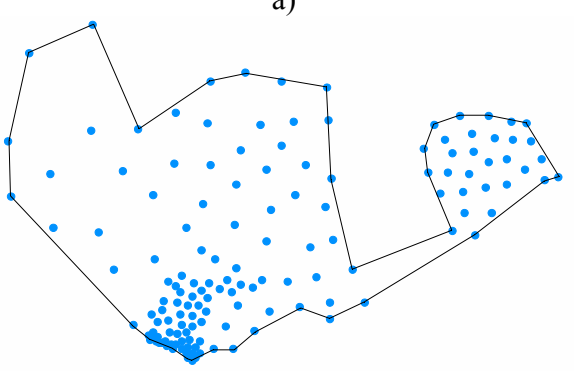

b)

Figure 7: Two hulls computed by the proposed algorithm.

Figure 7a shows a case where the shape of the region occupied by the points is very irregular. For this data set, a value of $k=5$ was used. The other case, in Figure 7b, illustrates the result obtained for a set of points with a large variation in the spatial density of the points and with two regions. In this example, the algorithm was started with $k=3$ but, in order to include the right-most group of points, the algorithm automatically increased the value of $k$ up to 18 . Both results were obtained with the lowest value of $k$ that permits the computation of the polygon. Using higher values of $k$ would lead to "smoother" polygons.

The proposed algorithm was already used in a real application that required the definition of geographic location contexts that are used to identify in which particular scenario a mobile user is located. The definition of the regions was done analysing a geographic database that integrates a total of 18914 POIs (Santos, 2006).

\subsection{Performance}

In order to evaluate the performance of the proposed algorithm in terms of computational load, the time used to compute the polygons was measured for several data sets of different sizes. The used test data sets were randomly generated within the space of a circle with unitary radius. For each data set, different values of $k$ were also used. Each point in the following graphs was obtained by averaging the several time values needed to process 20 different 
data sets. The obtained results are shown in Figure 8 and Figure 9.

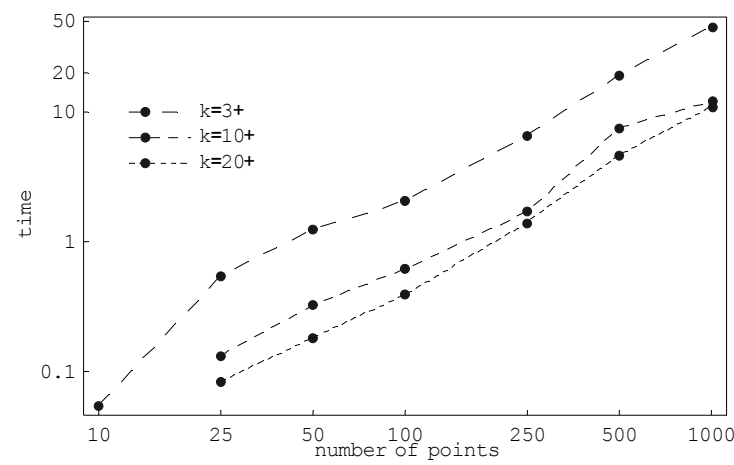

Figure 8: Time to compute the polygons vs. the number of points.

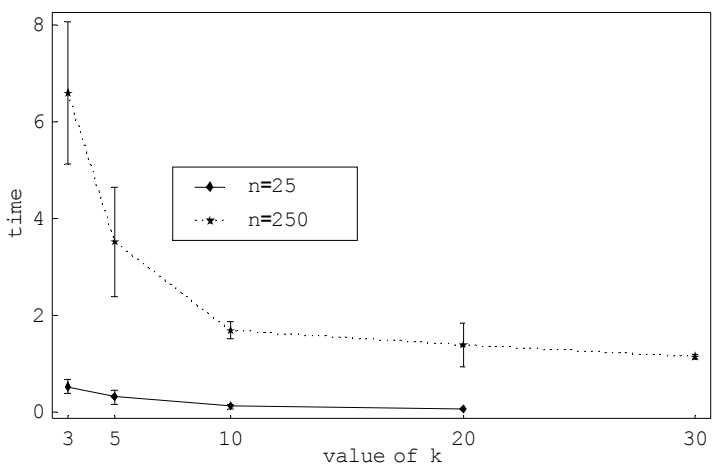

Figure 9: Time to compute the hull vs. the value of $k$.

In these graphs, the absolute values of the time used to compute the polygons is of less importance, since they depend on the used computer. Instead, these results are intended to assess the trends in the computing load when some parameters are changed. Moreover, these results were obtained from a Mathematica implementation of the algorithm, that has not been optimised for speed. The results presented here were obtained by running the algorithm in an ordinary Pentium 4-M at 2,2 $\mathrm{GHz}$ with 768 Mbytes of RAM.

Figure 8 shows the time (in seconds) used to compute the hulls for data sets of size 10, 25, 50, $100,250,500$ and 1000 points. The upper line represents the time values obtained when the algorithm was started with $k=3$. The lower lines represent the time values when the algorithm was started with $k=10$ and $k=20$, respectively. However, in the three cases and for some of the data sets, the algorithm recursively increased the value of $k$ to go around the special cases described in subsection 3.1. These results show that the time to compute the polygons increases approximately linearly with the number of points (note the log-log scales used in the graph).

The other result is that the computing time is smaller for higher values of $k$. This can be explained by the fact that, by starting with a higher $k$ (e.g. $k=20$ ), the time to try lower values of $k$ (e.g. 3 to 19 ) that might lead to special cases is removed from the total time. This is better shown in Figure 9, where the time to compute the hulls is shown as a function of the initial value of $k$, for $k=3,5,10,20$ and 30 , for two different sizes of the data set ( 25 and 250 points each). Here it is clear that lower values of $k$ result in higher computing times. Figure 9 also shows the standard deviation on the time taken to compute the different 20 data sets for each value of $k$. Here, the general trend is to observe a lower variation for higher values of $k$ than for lower values.

\subsection{More General Assessment}

Using the criteria defined in (Galton, 2006), and the same nomenclature where $S$ denotes the given set of points and $R(S)$ refers to the proposed region representing those points, the "concave hull" can be described as follows:

1. Outliers are not permitted, meaning that all the points of $S$ are within the computed polygon.

2. There are always points of $S$ on the boundary of $R(S)$.

3. The computed "concave hull" (polygon) is topological regular (unless the points are collinear).

4. The "concave hull" is connected.

5. The "concave hull" is polygonal.

6. The boundary of the "concave hull" is a Jordan curve (unless the points are collinear).

7. In some cases, such as in region D in Figure 3 , large areas of empty space are excluded from the "concave hull", unless a very large value for $k$ is used. In other cases, such as the one shown in Figure 7b, the large area of empty space in the upper-left region of the data set is maintained within the computed polygon.

8. The generalization of the Concave Hull algorithm to three dimensions might be possible, but not easily.

9. The analysis of the computational complexity of the Concave Hull algorithm is still future work.

Comparison of the Concave Hull algorithm with the SA algorithm described in (Galton, 2006) resulted in the following advantages of the Concave Hull. First, the use of the Concave Hull does not require any previous knowledge of the data set in 
order to choose the value of $k$. Starting the algorithm with $k=3$ always leads to a polygon with the characteristics described in the above criteria. On the other hand, if the SA algorithm is started with a too low value for $r$, the result may not be a regular polygon. Therefore, the choice of $r$ for SA requires a previous knowledge of the data set. This characteristic of the Concave Hull makes it suitable to process many data sets representing different regions, and where the spatial density of points in each region can be very different. Second, the Concave Hull algorithm adapts itself to the variations in the spatial density of the points within the same data set, as shown in Figure $7 \mathrm{~b}$. On the other hand, it seams that the SA algorithm uses a constant value of $r$ to select the list of candidates to become the next vertex of the polygon, therefore not being able to adapt to variations in the spatial density of the points.

\section{CONCLUSIONS}

In this paper we described an algorithm to compute the "concave hull" of a set of points in the plane. The algorithm is based in a $k$-nearest neighbours approach and is able to deal with arbitrary sets of points by taking care of a few special cases. The "smoothness" of the computed hull can also be controlled by the user through the $k$ parameter.

The presented algorithm has as advantages the fact that it can deal with non-convex (concave) hulls as well as convex ones, and the fact that the user can adapt the polygons to its needs by choosing the $k$ parameter. The algorithm was implemented as a Mathematica package, and the obtained results show that the time to compute the "concave hull" increases approximately linearly with the number of points.

Future work on this subject includes the improvement of the algorithm implementation, namely through the use of a more efficient function to calculate the angles depicted in Figure 5, and a more efficient function to verify if two line segments intersect each other. The computational complexity of the proposed algorithm is also a subject for future analysis.

\section{ACKNOWLEDGEMENTS}

This work was developed as part of the LOCAL project funded by the Fundação para a Ciência e
Tecnologia through grant POSI/CHS/44971/2002, with support from the POSI program.

\section{REFERENCES}

Graham, R.L., 1972, An efficient algorithm for determining the convex hull of a planar set, Information Processing Letters 1, 132-133

Jarvis, R.A., 1973, On the identification of the convex hull of a finite set of points in the plane. Information Processing Letters 2, 18-21

Preparata, F.P., and Hong, S.J., 1977, Convex hulls of finite sets of points in two and three dimensions. Communications of the ACM, 20, 2 (Feb.), 87-93.

Eddy, W.F., 1977, A new convex hull algorithm for planar sets. ACM Transactions on Mathematical Software, 3 , 4 (Dec.), 398-403.

Edelsbrunner, H., Kirkpatrick D.G, and Seidel R., 1983, On the Shape of a Set of Points in the Plane, IEEE Transactions on Information Theory, Vol. IT-29, No. 4, July

Galton, A, and Duckham, M., 2006, What is the Region Occupied by a Set of Points?, Proceedings of the Fourth International Conference on Geographic Information Science - GIScience 2006, Munich, Germany, September 20-23

Edelsbrunner, H., 1992a, Weighted Alpha Shapes, Technical Report: UIUCDCS-R-92-1760

Edelsbrunner, H., and Mucke, E.P., 1992b, Threedimensional Alpha Shapes, In Proceedings of the 1992 Workshop on Volume visualization, p.75-82, Boston, Massachusets, USA, October 19-20

Amenta, N., Bern, M., Kamvysselis, M., 1998, A New Voronoi-Based Surface Reconstruction Algorithm, Proceedings of the 25th annual conference on Computer graphics and interactive techniques, p.415421, July

LOCAL, 2006, http://get.dsi.uminho.pt/local, visited December 2006.

Santos, M. Y., and Moreira A., 2006, Automatic Classification of Location Contexts with Decision Trees, Proceedings of the Conference on Mobile and Ubiquitous Systems - CSMU 2006, p. 79-88, Guimarães, Portugal, June 29-30

Ertoz, L., Steinbach, M. and Kumar, V., 2003, Finding Clusters of Different Sizes, Shapes, and Densities in Noisy, High Dimensional Data. In Proceedings of the Second SIAM International Conference on Data Mining, San Francisco, CA, USA, May

Mathematica, http://www.wolfram.com, visited October 2006. 